This is the accepted manuscript, which has been accepted by IEEE for publication (C) 2014. Personal use of this material is permitted. Permission from IEEE must be obtained for all other uses, in any current or future media, including reprinting/republishing this material for advertising or promotional purposes, creating new collective works, for resale or redistribution to servers or lists, or reuse of any copyrighted component of this work in other works. The full reference is:

"Probabilistic Risk Assessment of Rotor Angle Instability Using Fuzzy Inference Systems"

R. Preece, J.V. Milanović

IEEE Transactions on Power Systems, vol. 30, issue 4, pp. 1747-1757, 2015

DOI: $10.1109 /$ TPWRS.2014.2352678 


\title{
Probabilistic Risk Assessment of Rotor Angle Instability Using Fuzzy Inference Systems
}

\author{
R. Preece, Member, IEEE, and J. V. Milanović, Fellow, IEEE
}

\begin{abstract}
This paper proposes a new method for the probabilistic risk assessment of rotor angle instability in power systems using fuzzy inference systems (FISs). The novel two-step approach first models the stochastic uncertainties present within the power system to produced probability density functions ( $p d f s$ ) for stability indicators. These stability indicators are established for both small and large disturbance rotor angle stability analysis. The $p d f s$ produced are subsequently decomposed into regions based on user-specified threshold values. The outputs from this decomposition are analyzed using fuzzy techniques to complete the risk assessment of instability. The methodology is applied to a multi-area test network into which a VSC-MTDC grid has been embedded to support power transfer from a number of large wind farms. This new combination of probabilistic and fuzzy techniques is shown to provide an effective methodology for quantifying the influence of system uncertainties on the risks of rotor angle stability.
\end{abstract}

Index Terms-Fuzzy set theory, large disturbance, risk analysis, rotor angle stability, small disturbance, stochastic uncertainty, VSC-MTDC.

\section{INTRODUCTION}

$\mathrm{R}$ ISK analysis is of growing importance in power systems research as operators seek to maximize the usage of existing assets without compromising system integrity. Power systems are increasingly operated in uncertain conditions as dependence on intermittent renewable energy sources grows and new load types are introduced. Risk analysis provides a framework in which these uncertainties and their potential consequences can be balanced against greater utilization of existing system assets.

The stochastic nature of these system uncertainties means that a probabilistic approach is required. Standard deterministic worst-case approaches fail to represent the expected variation and can be overly conservative - sometimes restricting asset usage under normal operation in case extremely rare multiple outage events occur. The use of fuzzy techniques to analyze the results of probabilistic studies allows rapid assessment of the risks associated with a given operating scenario. The fuzzy risk assessment can be easily tailored to the regulations and requirements of any power system. Furthermore it is able to provide meaningful risk values within

This work was supported by the Research Councils UK, through the HubNet consortium (grant number: EP/I013636/1).

R. Preece and J.V. Milanović are with the School of Electrical and Electronic Engineering, The University of Manchester, PO Box 88, Manchester, M60 1QD, UK. (email: robin.preece@manchester.ac.uk, milanovic@ manchester.ac.uk). user-defined ranges whilst maintaining enough detail to differentiate between scenarios which result in similar risk.

Fuzzy set theory has been applied to many areas of power systems studies [1] with much of the work focused on the effective control of system stabilizing devices without the need for detailed system models such as [2]-[4], and the commitment of generation considering uncertainties as in [5], [6]. Fuzzy techniques are able to deal with inherent uncertainty present when using linguistically fuzzy terms. This is beneficial when performing risk analysis, as Fuzzy Inference Systems (FISs) can handle uncertain fuzzy inputs but return crisp numerical risk values.

There has been limited application of fuzzy techniques to the stability assessment (and not stabilizing control) of power systems. Fuzzy classification techniques have been demonstrated as an effective means of detecting transient stability issues following the occurrence of a disturbance [7] and using steady state data [8]. Rotor angle stability to both small and large disturbances is critical to the successful operation of power systems and must be assessed thoroughly as system uncertainties increase. Fuzzy techniques can be used to complete this assessment and to quantify the risks involved in the operation of uncertain power systems. The application of FISs for a similar task is demonstrated in [9] where a dynamic security index for the system is calculated. However this approach uses deterministic values and does not consider the probabilities of events or calculate system risk levels.

The topic of risk assessment of small-disturbance stability issues has received very limited attention with most contributions acknowledging the need for probabilistic approaches such as [10]-[14] but falling short of full risk analysis. These works develop the use of probabilistic methods to establish statistical distributions of critical oscillatory system modes. However, they fail to evaluate risk in terms of both probability and severity of the resultant conditions - instead determining the probability of instability, or of critically underdamped oscillations. Some initial work in this area has been published by the authors in [15] in which it was shown that risk measures can be formulated to assess small-disturbance stability issues.

This paper proposes a novel two-step methodology that first simulates the effects that stochastic variations within the power system have on stability indicators. These stability indicators are established for both small and large disturbance rotor angle stability analysis. The $p d f s$ produced following Monte Carlo simulation are subsequently decomposed into regions based on user-specified threshold values. The outputs 
from this decomposition are analyzed using fuzzy techniques to complete the risk assessment of instability.

This research presents a probabilistic risk-based approach towards rotor angle stability assessment of power systems. Fuzzy inference systems are used to quantify both small and large disturbance rotor angle instability risks. The methodology presented is expandable and could be extended to multiple facets of stability and security analysis within power systems research. The proposed method is intended to complement traditional deterministic security assessment techniques by probabilistically handling the uncertainties inherent in power system operation. This method can be used to assess the risks associated with complex evolving systems that traditional deterministic approaches are unable to capture.

It is demonstrated that the use of cascading FISs allows the combination of multiple fuzzy risk values (FRVs) into a single system risk value. Furthermore, the use of fuzzy set theory allows an easily understandable and customizable approach whereby threshold values and performance criteria can be tailored to the system being studied and the regulatory framework under which it is operated. In this way, the applicability of the proposed approach is ensured.

\section{Probabilistic Fuzzy Risk Assessment Methodology}

The probabilistic fuzzy risk assessment methodology proposed can be considered as a two-step approach. First, the probabilistic distributions of critical system stability indicators through accurate modeling of stochastic uncertainties are produced. Second, fuzzy techniques are applied in order to analyze these $p d f s$ and translate them into meaningful risk values. Both stages of the approach are thoroughly described.

Many risk assessment approaches use economic measures to quantify the severity of events in order to facilitate direct comparisons. The mitigation of rotor angle stability issues within power systems in general can be a very demanding task. With many control schemes and protection devices installed to prevent system instability and consequently catastrophic events, correctly apportioning the economic valuation of any system collapse that does occur to these individual schemes is extremely complex. Therefore, the severity of stability issues is quantified using technical measures and probabilistic system stability indicators.

\section{A. Probabilistic System Stability Indicators}

The production of the $p d f s$ for system stability indicators is completed by modeling the stochastic variation of uncertain elements within the power system. A large number of feasible operating conditions are randomly generated which are subsequently investigated so that the stability indicators can be determined. Within this work, a numerical Monte Carlo (MC) approach is used to produce the pdfs. Efficient sampling techniques, such as the probabilistic collocation method [16] or two point estimation method [17], could be used to generate these $p d f s$ along with distributed processing approaches if greater computational efficiency is required.

Modern power systems are extremely reliable and the probability of experiencing stability issues within a given forecast horizon is very low. For this reason, pure MC simulation may fail to accurately produce $p d f$ tail regions and may miss low probability events entirely. A combination of MC simulation weighted by contingency probabilities will help ensure accurate $p d f$ generation. MC simulation can be used to account for continuously varying system uncertainties (such as operating conditions) whilst specific contingencies (for example, generator outages) can be weighted by their probability of occurrence. Additionally, methods such as importance sampling can be used to produce only the tail regions of distributions to reduce the computational burden of these studies if desired. Note that in this paper, the test system used displays much more marginal stability that would be the case in some practical systems. Contingencies are not considered in this illustrative example, and so only MC simulations are required to produce the $p d f s$.

The power system can be considered to consist of a set of uncertain system parameters. The set considered in this study includes bus loading levels, load power factors, generation output and power flow through HVDC lines. In addition to these system uncertainties, fault specific uncertainties must be considered when assessing the risk of transient disturbance stability issues. In this work, these include fault location (both the specific line and the position along the line) and the fault type (single-phase-to-ground, phase-to-phase, phase-tophase-to-ground, or three-phase). Further uncertainties that could be considered include the types of connected load and the resulting dynamic behavior of these loads and variation in system topology. Wherever possible, historical data for the power system in question should be utilized to accurately inform the stochastic modeling of each uncertain parameter.

\section{1) Small Disturbance Stability Indicator}

The damping of the critical electromechanical oscillatory mode is selected as the indicator for small disturbance instability of the power system. At each randomly selected operating point, the system model is linearized and eigenvalue analysis is used to identify the critical mode $\lambda_{\text {crit }}=\sigma_{\text {crit }} \pm j \omega_{\text {crit }}$. The settling time $T_{S}$ of the oscillations described by this secondorder response is inversely proportional to the damping ( $\sigma_{\text {crit }}$ ) of the mode with positive values representing small disturbance instability. The $p d f$ of $\sigma_{c r i t}$ is therefore used as the basis for the small disturbance fuzzy risk analysis.

\section{2) Large Disturbance Stability Indicator}

Large disturbance stability is assessed by the critical clearing time (CCT) of the system. A conditional $p d f$ of the CCT can be produced - conditional on the fact that a fault of some description has occurred. Different methods can be used to identify the CCT of a large multi-machine power network [18]. The extended equal area criterion [19] is one of the most often used methods. This method however, has limited application beyond first-swing unstable transient events. Timebased simulations on the other hand, ensure accurate modeling of system dynamics and can be performed iteratively to identify the conditional CCT. Though computationally much more intensive, their performance can be significantly enhanced by the use of variable time step integration, fast 
instability detection to interrupt simulations, and efficient search algorithms.

Iterative transient simulations are used within this work. Both operating conditions and fault details are generated randomly by considering the known stochastic variations of the uncertain parameters and event details. The MC process is used to produce a $p d f$ of the conditional system CCT that can subsequently be used for transient disturbance fuzzy risk analysis. It should be noted that the use of this conditional CCT will result in $p d f s$ with probabilities that may appear misrepresentative of true power system behavior. If the probability of the fault occurring in the time horizon being studied were also taken into account, the final probabilities considered would be much lower.

\section{B. Risk Assessment Using Fuzzy Techniques}

Fuzzy techniques are able to handle linguistically fuzzy terms, yet still provide crisp numerical outputs that can be used to quantify risk and guide system operation. They provide a good balance between standard risk matrices and continuous severity functions. Risk matrices are readily understandable but suffer from a lack of fine granularity due to the discrete nature of the outputs. Continuous severity functions can provide excellent differentiation between the risk values of various scenarios but are complex and require accurate definition to ensure correct risk quantification. A Fuzzy Inference System (FIS) is able to translate linguistically defined inputs, via easily defined if-then rules, into numerically crisp outputs within a user-defined range - providing both simplicity and fine granularity.

An FIS can be used to analyze the results from the probabilistic analysis and provide a Fuzzy Risk Value (FRV). The inputs for the Small Disturbance (SD-) FIS and Large Disturbance (LD-) FIS are sourced from the stability indicator $p d f s$. These $p d f s$ are decomposed based on predefined threshold values in order to determine the probability of the stability indicator falling within a given range. These ranges are defined for both SD and LD stability indicators.

\section{1) SD-FIS Input Definition}

Threshold values for $\sigma_{\text {crit }}$ (the small disturbance stability indicator) are chosen based on the settling time of electromechanical oscillations. The settling time $T_{S}$ is dependent on the tolerance (tol.) and $\sigma_{c r i t}$ according to (1). Equation (1) also shows the numerical result if, for example, a 5\% tolerance of the maximum deviation is desired (as in this work).

$$
T_{S}=\frac{\ln (\text { tol. })}{\sigma_{\text {crit }}}=\frac{\ln (0.05)}{\sigma_{\text {crit }}}=\frac{-3.00}{\sigma_{\text {crit }}}
$$

Threshold values are selected to decompose the $\sigma_{c r i t} p d f$ into regions representing oscillations which are:

- Unstable $\left(U_{S D}\right)$ : oscillations do not settle, $\left(\sigma_{\text {crit }}>0\right)$.

- Critical $\left(C_{S D}\right): T_{S}>60 \mathrm{~s},\left(-0.05<\sigma_{\text {crit }} \leq 0\right)$.

- Long lasting $\left(L_{S D}\right): 20<T_{S} \leq 60 \mathrm{~s},\left(-0.15<\sigma_{\text {crit }} \leq-0.05\right)$.

- Short lasting $\left(S_{S D}\right): T_{S} \leq 20 \mathrm{~s},\left(\sigma_{\text {crit }} \leq-0.15\right)$.

These regions can be user-defined by power systems engi- neers to accurately reflect the specific network and regulations under which it is operated. The values used in this work are for illustrative purpose only. The calculated probabilities for each region are used as inputs to the SD-FIS.

\section{2) LD-FIS Input Definition}

Large disturbance stability is indicated by the system CCT. Threshold values for this are selected based on typical protection device operation times taken from the Alstom Grid Network Protection \& Automation Guide [20]. If the CCT is extremely short, there is small chance that protection devices will be able to clear the fault fast enough to avoid system instability. Conversely, if the CCT is very long, many different graded protection devices will have sufficient time to operate, significantly reducing the risk of generators losing synchronism. The $p d f$ of the system CCT is therefore decomposed into the following regions:

- Very Short $\left(V_{L D}\right)$ : CCT $\leq 60 \mathrm{~ms}$, faster than typical operation times for primary protection devices.

- Short $\left(S_{L D}\right): \quad 60<\mathrm{CCT} \leq 300 \mathrm{~ms}$, reliant on successful operation of primary protection to clear the fault.

- Moderate $\left(M_{L D}\right): 300<\mathrm{CCT} \leq 850 \mathrm{~ms}$, time for backup protection in case of failure of primary protection.

- Long $\left(L_{L D}\right):$ CCT $>850 \mathrm{~ms}$, time for multiple protection schemes to operate before synchronism is lost.

As with calculation of the SD-FIS inputs, the thresholds for the CCT $p d f$ decomposition should be tailored to the system being evaluated and the protection schemes in use. Also note that additional decomposition zones can be included if required for the system in question. If this is the case, then additional FIS if-then rules should also be defined. It should be noted that in this work only rotor angle stability is of interest when determining the system CCT for each fault, despite the potential existence of other undesirable system phenomena (such as over-currents or under-voltages).

\section{3) FIS Input Fuzzification}

The probability of the stability indicators taking a value within the defined regions $\left(U_{S D}, C_{S D}, L_{S D}\right.$, and $S_{S D}$ for the SDFIS, and $V_{L D}, S_{L D}, M_{L D}$, and $L_{L D}$ for the LD-FIS) are determined for each FIS. These probabilities are then fuzzified using the input membership functions presented in Fig. 1. Triangular and trapezoidal functions are selected for simplicity, defining the membership $(\mu)$ of the probability values to five fuzzy sets: Extremely Rare (E) [0-1\%], Rare (R) [0-5\%], Infrequent (I) [1-15\%], Probable $(P)$ [10-90\%], and Frequent (F) [80-100\%]. This selected combination ensures even transitions between adjacent fuzzy states for the selected probability ranges. Other functions (such as Gaussian or sigmoid) and the probability ranges themselves can all be adjusted as desired by the user.

As with stability indicator range selection (e.g., oscillation settling time or critical clearing time), it is recommended that operational policy and regulatory documents are used to guide the selection of probability ranges. This forms an essential part of all risk analysis and is not unique to the use of a fuzzy approach. If continuous severity indices or risk matrices are used, it is still required to assign importance to probability 
values. The major benefit and flexibility offered by using a fuzzy approach is that these assignments are not crisp, i.e., not completely black and white. The grey fuzzy region describing transition between crisp regions is described mathematically and is actively used during the risk analysis procedure. Therefore, if system operators have less faith in the probability range boundaries that are defined, which is often the case, they can exploit the fuzziness (by increasing the region of overlap) to account for any uncertainty in the risk definition. The fuzzy approach therefore, reduces the influence of the selected probability ranges on the final risk value by keeping boundaries fuzzy and ultimately provides more robust results.

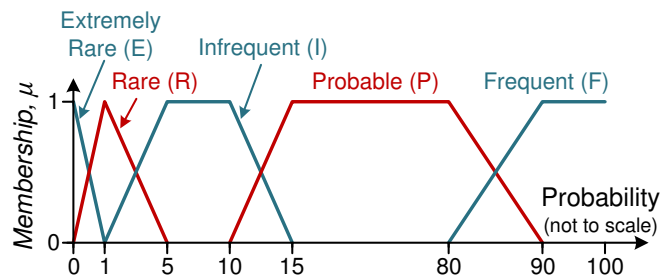

Fig. 1. FIS input membership functions for stability indicator range probabilities.

\section{4) Fuzzy Risk Evaluation}

Fuzzification of the inputs (or antecedents) provides the membership of each stability indicator region to the fuzzy probability sets shown in Fig. 1. A Mamdani-type FIS [21] is used to complete the fuzzy risk assessments of rotor angle stability. Each FIS evaluates a set of if-then rules using weighted implication methods in order to determine individual output (or consequent) functions. The consequent functions are subsequently aggregated together, before a defuzzification technique is used to provide a crisp fuzzy risk value (FRV).

TABLE 1

IF-THEN RULES FOR THE SD-FIS

\begin{tabular}{clcl}
\hline Rule & If & $\begin{array}{l}\text { Then } \\
\text { SD-FRV }\end{array}$ & Weight \\
\hline 1 & $\left(U_{S D}=F\right) \cup\left(C_{S D}=F\right)$ & High & 1 \\
2 & $\left(U_{S D}=P\right) \cup\left(C_{S D}=P\right)$ & High & 1 \\
3 & $\left(U_{S D}=I\right)$ & High & 1 \\
4 & $\left(L_{S D}=F\right)$ & Medium & 0.1 \\
5 & $\left(L_{S D}=P\right)$ & Medium & 0.1 \\
6 & $\left(C_{S D}=I\right)$ & Medium & 0.1 \\
7 & $\left(U_{S D}=R\right)$ & Medium & 0.1 \\
8 & $\left(S_{S D}=F\right)$ & Low & 0.01 \\
9 & $\left(S_{S D}=P\right)$ & Low & 0.01 \\
10 & $\left(L_{S D}=I\right) \cup\left(S_{S D}=I\right)$ & Low & 0.01 \\
11 & $\left(C_{S D}=R\right) \cup\left(L_{S D}=R\right) \cup\left(S_{S D}=R\right)$ & Low & 0.01 \\
12 & $\left(U_{S D}=E\right) \cup\left(C_{S D}=E\right) \cup\left(L_{S D}=E\right) \cup\left(S_{S D}=E\right)$ & Low & 0.01 \\
\hline & & & \\
\end{tabular}

TABLE 2

IF-THEN RULES FOR THE LD-FIS

\begin{tabular}{clcl}
\hline Rule & If & Then & Weight \\
& & LD-FRV & \\
\hline 1 & $\left(V_{L D}=F\right) \cup\left(S_{L D}=F\right)$ & High & 1 \\
2 & $\left(V_{L D}=P\right) \cup\left(S_{L D}=P\right)$ & High & 1 \\
3 & $\left(V_{L D}=I\right)$ & High & 1 \\
4 & $\left(M_{L D}=F\right)$ & Medium & 0.1 \\
5 & $\left(M_{L D}=P\right)$ & Medium & 0.1 \\
6 & $\left(S_{L D}=I\right)$ & Medium & 0.1 \\
7 & $\left(V_{L D}=R\right)$ & Medium & 0.1
\end{tabular}

\begin{tabular}{clll}
8 & $\left(L_{L D}=F\right)$ & Low & 0.01 \\
9 & $\left(L_{L D}=P\right)$ & Low & 0.01 \\
10 & $\left(M_{L D}=I\right) \cup\left(L_{L D}=I\right)$ & Low & 0.01 \\
11 & $\left(S_{L D}=R\right) \cup\left(M_{L D}=R\right) \cup\left(L_{L D}=R\right)$ & Low & 0.01 \\
12 & $\left(V_{L D}=E\right) \cup\left(S_{L D}=E\right) \cup\left(M_{L D}=E\right) \cup\left(L_{L D}=E\right)$ & Low & 0.01 \\
\hline
\end{tabular}

The rule set for the SD-FIS is presented in Table 1, and for the LD-FIS in Table 2. A shorthand notation is used so that small-disturbance unstable oscillations are frequent is denoted by $\left(U_{S D}=F\right)$, based on the relevant letters in parentheses throughout this methodology. For both FISs, weights are used to ensure that High FRVs take precedence over Medium FRVs (which subsequently take precedence over Low FRVs). The weights shown in Table 1 have been selected from a number of tested candidate options as they ensure the hierarchy of individual consequent values is preserved in the final defuzzified FRV. The use of weights also reduces the total rule set as not all possible input permutations must be defined. Membership functions for the consequent FRV fuzzy sets are shown in Fig. 2. Initial results using symmetrical triangular membership functions resulted in poor differentiation between results within the same final risk categorization (Low, Medium, or High). Skewed triangular sets have therefore been selected to ensure that variations in membership values will result in variations in the final defuzzified FRV.

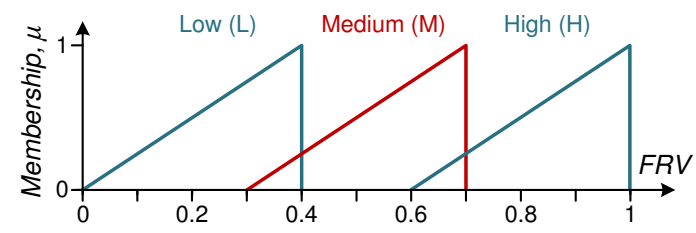

Fig. 2. Output fuzzy risk value (FRV) membership functions.

A variety of logical operators, implication methods, aggregation methods and defuzzification techniques can be applied with fuzzy inference systems [21]. Within this work, a maximum function is applied for or operations, the implication method is the minimum, the aggregation method is a sum, and centroid defuzzification is used. These common Mamdani inference rule base operations [22] provide standard extensions of Boolean logic and have demonstrated good results.

It must be stressed that the fuzzification membership functions and the rule sets can and should be tailored to meet the requirements of practical systems which will operate under different security criteria. For example, if desired, additional output FRV functions can be described to account for high impact events such as blackouts, with rules appropriately weighted to ensure that even a low probability of occurrence for an event will not mask its significance.

\section{TEST NETWORK \& SYSTEM UNCERTAINTIES}

The methods described within this paper are illustrated using a heavily modified version of the 16 machine, 68 bus reduced order representation of the New England Test System and the New York Power System (NETS \& NYPS). The network (including modifications) is shown in Fig. 3. System analysis and simulations are all performed within the MATLAB/Simulink environment making use of modified 
MATPOWER [23] functions to perform optimal power flows.

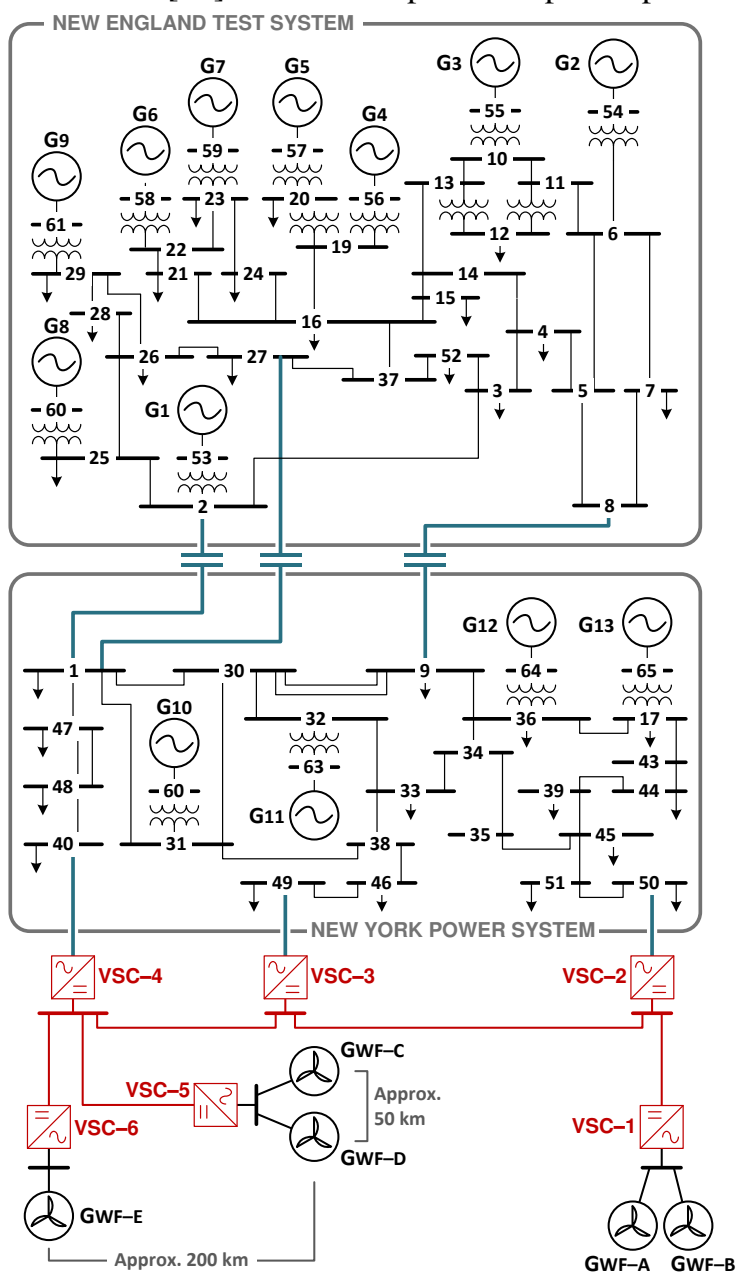

Fig. 3. Modified NETS NYPS test network, including six-terminal VSCMTDC grid and additional wind farms.

\section{A. AC System Details}

Generators G1-13 are modeled and controlled as detailed in [24] with G1-8 under slow DC excitation (IEEE-DC1A), G9 equipped with a fast acting static exciter (IEEE-ST1A) and a Power System Stabilizer (PSS), and G10-13 under constant manual excitation. All generators are represented by full sixth order models. System loads are modeled as constant impedance. Other load models can be used without any loss of generality of the proposed methodology. Full system details, generator and exciter parameters are given in [24] with PSS settings for G9 taken from [25].

The standard test network in [24] also contains three large generators representing external networks which import approximately $2.1 \mathrm{GW}$ into the NYPS area under nominal loading. In the modified network, the generators, buses, and lines composing these external networks have been replaced with a five line, six terminal VSC-MTDC network connected to five $450 \mathrm{MW}$ wind farms (GWF-A-E).

Additionally, due to the integration of a large amount of intermittent generation, the inter-area $\mathrm{AC}$ ties between the NETS and NYPS region have been compensated with series capacitors. The compensation of these lines (line 1-2, line 127, and line 8-9) allows increased power transfer from NETS to NYPS during periods when wind generation is low.

\section{B. VSC-MTDC System Details}

The VSC-MTDC system enables power transfer from the wind farms into the AC network. Each converter station is modeled as an injection of active and reactive power neglecting high frequency device switching operations [26]. Converter station controllers are included as described in [27] and DC lines are modeled as presented in [28].

All converter stations connected to the NYPS region ( $V S C$ 2-4) regulate active power injection into the AC system using a $D C$ voltage droop characteristic to facilitate power sharing during HVDC outage contingencies [29]. Additionally, VSC2-4 all support the local AC voltage at the connection bus through reactive power injection. This control is set so that reactive power injection will not vary unless local AC system voltages vary outside a deadband of $\pm 0.05 \mathrm{pu}$ around the voltage reference (the initial load flow solution). These converters, therefore, operate in Active Power Voltage Droop-AC Voltage control mode.

The active power injected into the MTDC system at each converter station connected to a wind farm $(V S C-1,5,6)$ is determined by the output of the renewable energy sources. Reactive power is supplied as required to support the renewable generation. These converters operate in AC Frequency-AC Voltage control mode. All controller and system details for the VSC-MTDC system are provided in Appendix I.

\section{Wind Farm System Details}

Five $450 \mathrm{MW}$ wind farms (GWF-A-E) are connected to the test network through the VSC-MTDC system. For the studies performed it has been assumed that the power output from the wind farm will be constant during each individual investigated operating point (i.e. wind speed fluctuations are neglected). As the converters connected to the wind farms operate in $A C$ Frequency-AC Voltage control, all power produced by the wind farm is transferred to the VSC-MTDC system.

\section{Operational Constraints}

An optimal power flow solution is used within this work to more accurately generate representative system operating points. All voltages are constrained within the range 0.9-1.1 pu. Generator cost data, and active and reactive power limits can be found in [30].

For practical implementations it is also important to include all known security constraints that exist within the network. This will typically include static constraints (e.g. line current limits), dynamic security proxies (e.g. inter-area power transfer limits to minimize oscillations) which may be expressed as nomograms. Inclusion of these will ensure that the $p d f s$ produced accurately represent the system variation due to uncontrollable system uncertainty and do not contain situations that would be avoided through mitigating control action.

Such constraints are not provided for this test system and to arbitrarily create them for this purpose would not be of any practical value. However, in order to anticipate how inclusion of such operational security constraints may affect the quantitative results obtained - a study is included in Section IV 
which addresses this and helps to illustrate the proposed methodology.

\section{E. System Uncertainties}

The uncertainty surrounding operational forecasts and intermittent generation will result in variations of the critical system stability indicators. This section will outline the uncertainties that are considered for the test system described.

\section{1) Loading Uncertainty}

Variation in the loading values and load power factors ( $p . f$.) at each AC system bus represent uncertainty in loading forecasts. The correlation of different load types is also considered with loads categorized as residential or industrial based on their nominal p.f. (values over 0.9 are classed as residential). Correlation coefficients $\rho$ between different loads are $\rho=0.8$ between residential loads, $\rho=0.4$ between industrial loads, and $\rho=0.2$ between residential and industrial loads [31]. All loads follow a Gaussian distribution with nominal mean values and standard deviation (s.d.) of $1.67 \%(5 \%$ at $3 \sigma$ ). Load p.f. values are non-correlated with s.d. of $1.67 \%$.

\section{2) Wind Farm Generation Uncertainty}

Electrical power output from the multiple wind farms (GAE) is determined by the local wind speed $v$. Within this work, $v$ is a random variable sampled from a Weibull distribution, as described by (2).

$$
f(v)= \begin{cases}\frac{k}{\varphi}\left(\frac{v}{\varphi}\right)^{k-1} e^{-(v / \varphi)^{k}} & \text { for } v \geq 0, \\ 0 & v<0 .\end{cases}
$$

In (2), $k$ is the shape parameter and $\varphi$ is the scale parameter (commonly signified by $\lambda$ but called $\varphi$ here to avoid confusion). In this study, values for these parameters were sourced from [32] with $k=2.2$ and $\varphi=11.1$. Each wind farm has a total generating capacity of $450 \mathrm{MW}$ and is assumed to consist of 90 Areva M5000 5 MW turbines [33]. The total power produced is calculated by generating the local wind speed, determining a single turbine's output according to its power curve, and then scaling the individual turbine output to the capacity of the whole wind farm. More accurate calculation of wind farm output e.g., [34], [35] is possible though this would not affect the methodology presented, only the final numerical results.

As multiple wind farms are connected to the same VSCMTDC converter stations, a level of correlation in the wind speeds they experience is expected. Correlated multivariate Weibull distributed random variables are modeled by initially generating marginal univariate distributions related by a multivariate Gaussian copula. These random variables are subsequently transformed into Weibull distributed parameters, preserving the rank correlation between the initial Gaussian variables.

The correlation coefficients used to define the Gaussian copula are sourced from [36] and it is assumed that wind farms connected to the same converter station are located 50 $\mathrm{km}$ apart, and wind farms connected to different converter stations are $200 \mathrm{~km}$ apart. These assumptions result in correlation coefficients of $\rho=0.73$ and $\rho=0.58$ for $50 \mathrm{~km}$ and 200 $\mathrm{km}$ separation respectively.

\section{3) VSC-MTDC Operational Uncertainty}

The VSC-MTDC grid operates to deliver all power from the intermittent wind resource to the AC network and therefore the uncertainty surrounding its operation is tied to the wind farm power output. A simple power sharing strategy is utilized within this study wherein VSC-2 delivers $40 \%$ of the total wind power production, VSC-3 delivers $20 \%$, and VSC-4 acts as a slack to account for the losses within the VSCMTDC system (approximately $2.5 \%$ of total power transmission). It should be noted that this slack behavior only occurs during the DC load flow solution and that for dynamic studies the voltage droop characteristic ensures that variations in active power injection are shared by all converters.

\section{4) Fault Specific Uncertainty}

For large disturbance stability risk assessment, the uncertainty surrounding the disturbance must also be considered in addition to system operating condition uncertainties. It is assumed that all faults occur on transmission lines, and not within transformers or on system buses. The specific line is selected randomly with a probability equal to the ratio between the impedance of the specific line and sum of all transmission line impedances within the network. The position fault position along the line follows a uniform distribution as in [37]. Different fault types have different probabilities of occurrence with a $70 \%$ probability of Line-Ground $(L-G)$, $15 \%$ probability of Line-Line $(L-L), 10 \%$ probability of LineLine-Ground $(L-L-G)$, and a $5 \%$ probability of Line-LineLine $(L-L-L)$ faults, adopted from [38]. Unbalanced faults are modeled as proper shunt admittances with equivalent fault admittances calculated as in [18].

\section{APPLICATION, RESUlts AND DISCUSSION}

The probabilistic risk assessment methodology using fuzzy techniques has been applied to the test system described in order to assess the risk of rotor angle instability issues. This has been completed by simulating the uncertainties described for both small and large disturbance stability analysis. This illustrative test system does not exhibit the same high levels of reliability as practical power systems as it does not contain parallel transmission paths and is a reduced order equivalent model. Therefore the probabilities of stability issues and associated risks will be higher for this illustrative example than anticipated for practical systems.

\section{A. Small Disturbance Risk Assessment}

The probabilistic fuzzy risk assessment has been completed with varying levels of installed series capacitor compensation on the inter-area AC ties between the NETS and NYPS regions (shown in Fig. 3). The modal plot for the critical system mode (of approximately $0.6 \mathrm{~Hz}$ ) following $5000 \mathrm{MC}$ runs when no compensation is installed is shown in Fig. 4. Also shown in Fig. 4 is the $p d f$ of $\sigma_{c r i t}$ (determined using a kernel smoothing density estimate) and the probabilities that critical mode oscillations will be short $\left(S_{S D}\right)$, long $\left(L_{S D}\right)$, critical $\left(C_{S D}\right)$, and unstable $\left(U_{S D}\right)$.

The resulting probabilities for the SD stability indicator 
ranges are used as the inputs for the fuzzy risk assessment. Fig. 5 demonstrates the rule evaluation process for the system with no compensation, where the four rules with non-zero consequents are displayed (refer to Table I for the symbols used in this figure). The consequent risk areas (selected according to each rule) are filled according to the membership level of the antecedent at the input (probability) value denoted by the dashed red lines. This consequent risk area is subsequently weighted before being aggregated together. The final defuzzified FRV is calculated as the centroid of the final aggregated risk area and is denoted by a further dashed red line. It is calculated to be 0.76 , and there is therefore high risk.

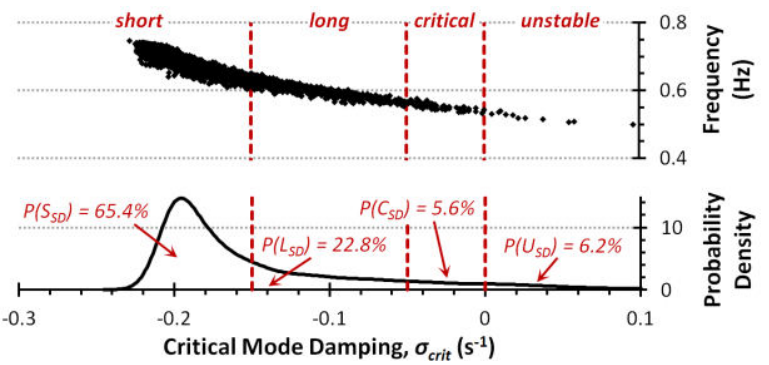

Fig. 4. Modal plot and resulting $p d f$ for critical mode damping with no series compensation.

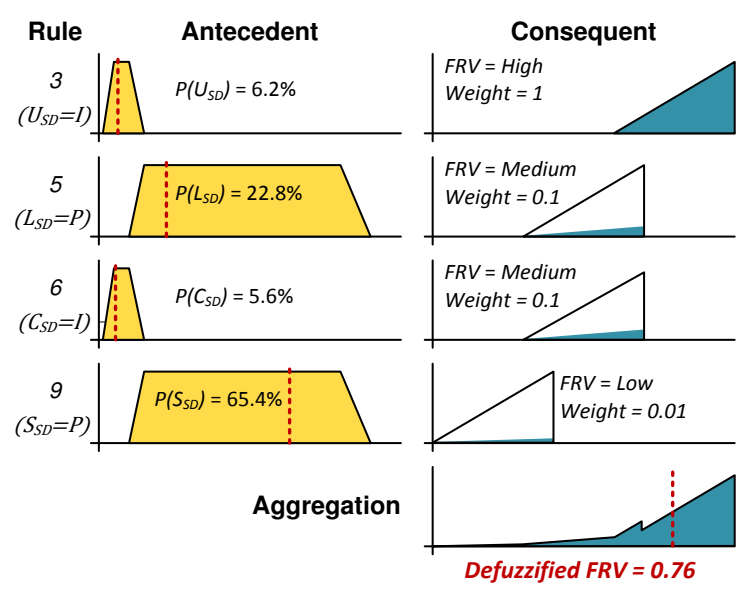

Fig. 5. SD-FIS rule evaluation with no series compensation installed.

Probabilistic fuzzy risk assessments have also been completed for the test system with $30 \%$ and $50 \%$ series compensation levels. The inclusion of the series capacitors aids power transfer from the NETS area to the NYPS area, reducing the stress on the network and resulting in fewer instances when oscillations are critical or unstable. This is clearly evident in Table 3, where critical oscillation region probabilities and final SD-FRV values are collated.

TABLE 3

SD-FRV VALUES WITH VARYING LEVELS OF SERIES COMPENSATION

\begin{tabular}{|c|c|c|c|c|c|}
\hline \multirow{2}{*}{$\begin{array}{c}\text { Level of Series } \\
\text { Compensation }(\%)\end{array}$} & \multicolumn{4}{|c|}{ Critical oscillation probabilities (\%) } & \multirow{2}{*}{$S D-F R V$} \\
\hline & $P\left(U_{S D}\right)$ & $P\left(C_{S D}\right)$ & $P\left(L_{S D}\right)$ & $P\left(S_{S D}\right)$ & \\
\hline 0 & 6.2 & 5.6 & 22.8 & 65.4 & 0.76 \\
\hline 30 & 0.3 & 1.4 & 17.2 & 81.1 & 0.46 \\
\hline 50 & 0 & 0.1 & 6.2 & 93.7 & 0.21 \\
\hline
\end{tabular}

1) Anticipated Effects of Security Constraints

As stated in Section III.D, this test network is simulated using very few operational constraints. For practical implementations (in real power systems) the inclusion of multiple static limits and dynamic security proxies would likely skew the $p d f s$ obtained during the probabilistic assessment towards more stable situations (with many marginally stable and unstable cases removed by the included constraints). As previously stated, these constraints are not available for this network as they simply do not exist. Nevertheless, it is possible to anticipate their effects - the pdf skewing - and to generate results to represent this. These results can then be used to further demonstrate the robustness of the proposed method (whilst it is acknowledged that the precise quantitative results would likely change for practical implementations).

Three example $p d f s$ have been generated using the generalized extreme value (GEV) distribution (which provides a good approximation of the distribution in Fig. 4). These are shown in Fig. 6 where the skewing effects can be seen, particularly on the $p d f$ during the (zoomed) unstable regions. Pdf $(a)$ is broadly analogous to the results obtained using the full study with no series compensation installed - with $p d f s(b)$ and $(c)$ representing increasingly more secure system operation. These example $p d f s$ have been analyzed using the SD-FIS to produce final fuzzy risk values shown in Table 4.

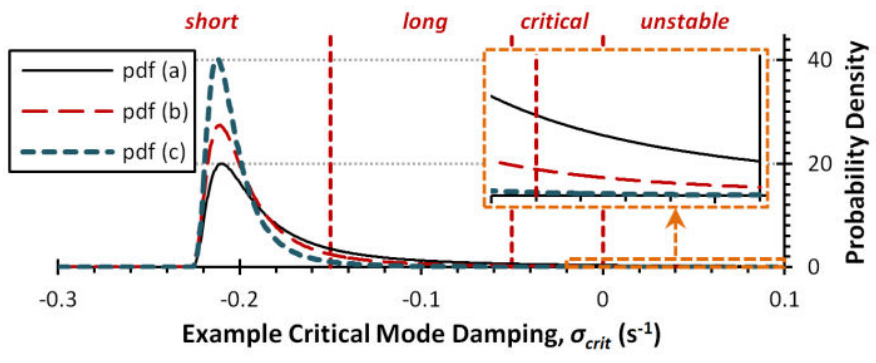

Fig. 6. Example $p d f$ s for critical mode damping from GEV distributions.

TABLE 4

SD-FRV VALUES FOR ANTICIPATED EFFECTS OF SECURITY CONSTRAINTS

\begin{tabular}{|c|c|c|c|c|c|}
\hline \multirow{2}{*}{$P d f$} & \multicolumn{4}{|c|}{ Critical oscillation probabilities (\%) } & \multirow{2}{*}{$S D-F R V$} \\
\hline & $P\left(U_{S D}\right)$ & $P\left(C_{S D}\right)$ & $P\left(L_{S D}\right)$ & $P\left(S_{S D}\right)$ & \\
\hline (a) & 5.6 & 2.3 & 15.1 & 77.0 & 0.78 \\
\hline (b) & 1.2 & 0.8 & 8.4 & 89.6 & 0.54 \\
\hline (c) & 0.1 & 0.1 & 2.6 & 97.2 & 0.28 \\
\hline
\end{tabular}

It is evident from these results that the SD-FRV values obtained from the nominally more secure pdfs result in reduced risk values. This is as would be expected from this robust fuzzy risk analysis methodology.

\section{2) Capturing High Impact Low Probability Events}

It has also been emphasized that this proposed fuzzy methodology can be easily customized to include further user defined risks. To illustrate this, an example is presented to demonstrate how risks associated with high impact low probability events can be included.

Assuming further analysis (such as contingency studies) can provide the probability of small-disturbance stability related blackout $P$ (Blackout), this can easily be incorporated into the existing SD-FIS. Fig. 7 shows new membership functions that can be used to include this feature. The probability membership function is concerned only with whether or 
not blackouts are possible and utilizes a logarithmic scale. The final fuzzy risk value has an extreme risk classification added to the $S D-F R V$ membership functions previously described by Fig. 2. In addition, the simple (highly weighted) rule in Table 5 is added to the SD-FIS to incorporate the risk associated with this high impact event.
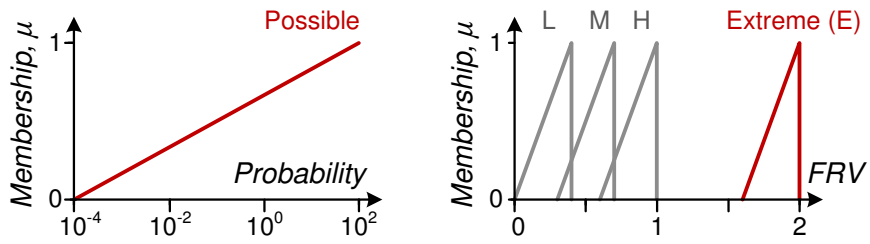

Fig. 7. Input probability (left) and output FRV (right) membership functions for blackouts.

TABLE 5

ADDITIONAL IF-THEN RULE FOR THE SD-FIS INCLUDING BLACKOUT

\begin{tabular}{cccc}
\hline Rule & If & Then $S D-F R V$ & Weight \\
\hline 13 & (Blackout $=$ Possible $)$ & Extreme & 10 \\
\hline
\end{tabular}

With these additional membership functions and rule 13 included, and assuming that $P($ Blackout $)=0.001 \%$, the new SD-FIS provides a fuzzy risk value of 1.37 . If the probability of blackout increases to $0.01 \%$ (i.e. by a factor of ten), the final FRV increases to 1.52. From Fig. 7, it is clear that any value of FRV greater than one will indicate the potential for high impact events and should be carefully investigated.

\section{B. Large Disturbance Risk Assessment}

The fuzzy risk assessment has also been completed for large disturbances. In all cases, faults are cleared through the removal of the faulted line. No auto-reclosing schemes are considered within this study, though they could be included as required by considering the probability of successful reclosing (on a per feeder basis if historical data allows).

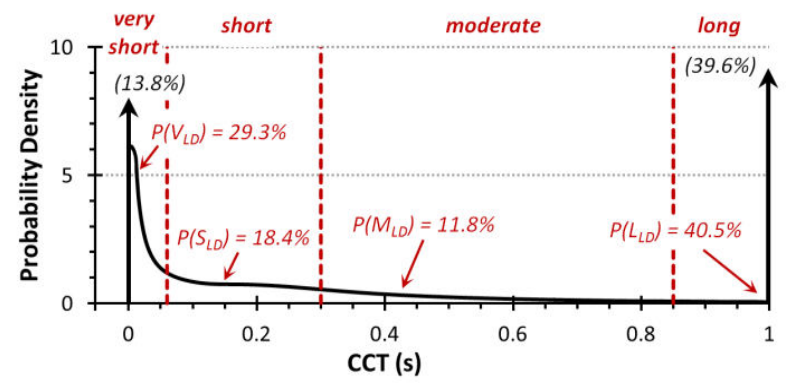

Fig. 8. Pdf for system CCT with no series compensation.

To ensure accurate representation of the faster time periods in the CCT $p d f, 1000 \mathrm{MC}$ runs for each fault type are simulated, before scaling and summing the individual $p d f s$ according to the fault-type probabilities previously stated. This ensures that the more serious, but less probable, fault types are simulated many times and reduces the risk of outlying results skewing the $p d f$. To improve total simulation time, any CCTs longer than $1 \mathrm{~s}$ are not determined and a CCT of $1 \mathrm{~s}$ is assumed for that given set of system uncertainties. The resulting $p d f$ of the system CCT (the large disturbance stability indicator) is shown in Fig. 8, when no compensation is used. Also shown, are the probabilities for the CCT regions: Very Short $\left(V_{L D}\right)$, Short $\left(S_{L D}\right)$, Moderate $\left(M_{L D}\right)$, and Long $\left(L_{L D}\right)$. The arrows represent lumped probabilities for CCTs of $0 \mathrm{~s}$ and $1 \mathrm{~s}$. $13.8 \%$ of cases will have a CCT of $0 \mathrm{~s}$ due to the criticality of the faulted line with its disconnection resulting in instability. The $39.6 \%$ of cases with CCT greater than 1s will follow the same exponential decay seen between $0-1 \mathrm{~s}$. However, resolution of this portion of the $p d f$ is not required for these studies and therefore has not been determined.

The probabilities shown in Fig. 8 are used as inputs to the LD-FIS to determine the fuzzy risk value. Rule evaluation is not shown but the final LD-FRVs are detailed in Table 6 for the system without and with same levels of capacitor series compensation considered as for the small disturbance analysis.

It is evident from Table 6 that the level of compensation has little effect on the LD-FRVs with high risk values of 0.80 or higher in all cases. The minor variations between the final fuzzy risk values are due to the variation caused by the MC process. The high risk of large-disturbance instability is caused by the topology of the network with many faults resulting in CCTs of $0 \mathrm{~s}$ (due to instability when the faulted line is cleared by disconnection of the affected line). In practical power systems, such critical lines would consist of double circuits to ensure that the loss of one line would not result in transient instability and the collapse of the system - significantly increasing the CCT. Nevertheless, it is evident that the presented methodology is readily applicable to both small and large disturbance rotor angle stability risk assessments.

TABLE 6

LD-FRV VALUES WITH VARYING LEVELS OF SERIES COMPENSATION

\begin{tabular}{|c|c|c|c|c|c|}
\hline \multirow{2}{*}{$\begin{array}{c}\text { Level of Series } \\
\text { Compensation }(\%)\end{array}$} & \multicolumn{4}{|c|}{ CCT probabilities (\%) } & \multirow{2}{*}{$L D-F R V$} \\
\hline & $P\left(V_{L D}\right)$ & $P\left(F_{L D}\right)$ & $P\left(M_{L D}\right)$ & $P\left(S_{L D}\right)$ & \\
\hline 0 & 29.3 & 18.4 & 11.8 & 40.5 & $\mathbf{0 . 8 3}$ \\
\hline 30 & 27.6 & 18.5 & 19.8 & 34.1 & 0.80 \\
\hline 50 & 30.9 & 19.2 & 10.8 & 39.1 & 0.84 \\
\hline
\end{tabular}

\section{FUZZY RISK OF SYSTEM RotoR ANGLE INSTABILITY}

The small and large disturbance fuzzy risk values can also be combined to determine a single Rotor Angle Stability (RAS) FRV. As with the previous risk assessments, this can be achieved using an FIS. The FISs are cascaded as shown in Fig. 9 in order to determine a single risk value that can be used to describe the exposure to instability due to the uncertainty surrounding the forecast operating point.

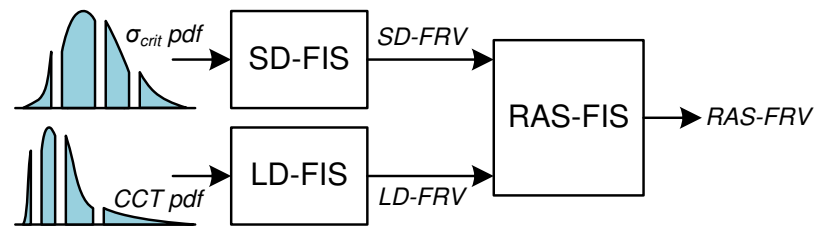

Fig. 9. Cascading fuzzy inference systems to produce a system level rotor angle stability fuzzy risk value (RAS-FRV).

The inputs to the RAS-FIS are the SD- and LD-FRVs (as determined using the previously outlined FIS structures). Input and output membership functions are given by the triangular functions previously described in Fig. 2. The rule set for this FIS is defined simply using the three if-then rules in Table 7. The differences between the rule weights are reduced for this 
FIS (compared to the previous FISs) so that the final RASFRV reflects a balance of the SD- and LD-FRVs - whereas previously the primary desire was to highlight any high risk level. The rule set is, however, still weighted towards prioritizing high risks, and these weights can (of course) be modified to reflect the risk evaluation performance desired.

Rule evaluation for the three system scenarios (with varying levels of series compensation) results in the final RASFRVs detailed in Table 8. It is evident that these risk values are dominated by the high large disturbance risk values.

TABLE 7

IF-THEN RULES FOR THE RAS-FIS

\begin{tabular}{clcl}
\hline Rule & If & $\begin{array}{l}\text { Then } \\
\text { RAS-FRV }=\end{array}$ & Weight \\
\hline 1 & $(S D-F R V=H) \cup(L D-F R V=H)$ & High & 1 \\
2 & $(S D-F R V=M) \cup(L D-F R V=M)$ & Medium & 0.4 \\
3 & $(S D-F R V=L) \cup(L D-F R V=L)$ & Low & 0.2 \\
\hline
\end{tabular}

In all three cases, the final rotor angle stability fuzzy risk value is high (with values always greater than 0.73 ). These values are dominated by the large disturbance risks which are high and unaffected by the level of series compensation. However, the differences between the small disturbance risk values affect the RAS-FRVs - with the risk reducing as the level of series compensation rises. It is evident from this example and Fig. 9 that cascading FISs can be used to quantify the system risk level using simple and understandable rules, whilst still maintaining a sufficient level of detail to differentiate between scenarios.

TABLE 8

RAS-FRV VALUeS WITH VARYING LEVELS OF SERIES COMPENSATION

\begin{tabular}{cccc}
\hline $\begin{array}{c}\text { Level of Series } \\
\text { Compensation (\%) }\end{array}$ & SD-FRV & LD-FRV & $\boldsymbol{R A S - F R \boldsymbol { V }}$ \\
\hline 0 & 0.76 & 0.83 & $\mathbf{0 . 8 5}$ \\
30 & 0.46 & 0.80 & $\mathbf{0 . 7 6}$ \\
50 & 0.21 & 0.84 & $\mathbf{0 . 7 3}$ \\
\hline
\end{tabular}

\section{A. Comparison with Risk Matrix Approach}

In order to highlight the benefits initially described in Section II.B - namely that fuzzy systems are described by simple and comprehendible rule sets and provide numerically crisp risk values - the results from the RAS-FIS are compared with those obtained using a traditional risk matrix approach.

The scarcity, if not complete lack, of methods for risk analysis of system stability hinders robust comparison of the proposed fuzzy approach with other approaches. Of the previously discussed work on fuzzy approaches and probabilistic stability assessment of power systems, [7]-[9] focus on purely deterministic application of fuzzy logic, whilst [10][14] demonstrate probabilistic analysis but do not attempt to quantify the severity of resultant conditions and therefore are not measures of risk. The new work in [15] demonstrates the application of risk analysis to small-disturbance stability using risk matrices to quantify the system risk level. The risk matrix approach represents an established risk quantification technique that has been utilized in many sectors and with which system operators are most familiar. It is therefore, used here, as the only suitable, if not only available, benchmark for the comparison with the proposed fuzzy approach.

The risk matrix shown in Fig. 10 is an equivalent representation of the RAS-FIS described by the weighted rule set in Table 7. Evaluation of the final combined rotor angle stability risk is completed and the results are presented in Table 9.

\begin{tabular}{|c|c|c|c|c|}
\hline & \multicolumn{3}{|c|}{$S D-F R V$} \\
\hline & & High & Medium & Low \\
\hline \multirow{3}{*}{$\begin{array}{l}\vec{\alpha} \\
\frac{1}{1} \\
\frac{1}{3}\end{array}$} & High & High & High & High \\
\hline & Medium & High & Medium & Medium \\
\hline & Low & High & Medium & Low \\
\hline
\end{tabular}

Fig. 10. Comparative risk matrix, equivalent to the RAS-FIS hierarchical rule set.

It is readily evident that the use of the risk matrix - whilst valuable for high level risk analysis - fails to preserve much of the information and offers no differentiation between different scenarios resulting in the same final risk classification. The fuzzy approach, however, is shown to provide valuable, numerical risk values which enable more informed operational decisions.

TABLE 9

COMPARISON OF RAS-FIS AND RISK MATRIX ANALYSIS

\begin{tabular}{ccc}
\hline Level of Series Compensation (\%) & RAS-FRV & Risk Matrix \\
\hline 0 & 0.85 & High \\
30 & 0.76 & High \\
50 & 0.73 & High \\
\hline
\end{tabular}

\section{CONCLUSIONS}

This paper presented a novel two-step methodology to quantify the risk of rotor angle instability within power systems. The proposed technique first simulates the stochastic variations within the power system using the Monte Carlo approach to determine how stability indicators (such as critical mode damping, or fault critical clearing times) are affected. The $p d f s$ for these stability indicators are decomposed based on user-defined threshold values to determine the probability that the stability indicator will exist within given ranges. These probabilities are used as the inputs to FISs which are used to assess the risk value by evaluating easily defined rule sets. Furthermore, these FISs can be combined to determine a single risk level for the system.

This novel approach to probabilistic risk assessment of rotor angle stability has been demonstrated using a multi-area power system model including a VSC-MTDC grid with large penetration of intermittent wind generation. The results have illustrated the effectiveness of combining fuzzy techniques with probabilistic analysis for risk assessment. Furthermore, the approach is readily customizable as threshold values and rule sets can be tailored to the system being studied and the regulatory framework under which it is operated. This methodology provides an expandable framework which can be used to assess further facets of risk assessment through the definition of suitable indicators. As with all risk analysis techniques, the final rule sets and inference systems used must 
be validated against offline contingency analysis in order to thoroughly evaluate the ability of the risk analysis technique to highlight and quantify issues arising in prescribed scenarios and operating conditions.

\section{APPENDIX I: VSC-MTDC SYSTEM \& CONTROLLER DATA}

All data provided is based on a 100 MW HVDC base (with $V_{D C}^{\text {base }}=320 \mathrm{kV}$ ). Converter stations cause active power flow losses of $1 \%$. The droop characteristic is defined as (3).

$$
P_{\text {droop }}^{\text {ref }}=P^{r e f}\left[1+k_{d r o o p}^{V d c}\left(V_{d c}^{r e f}-V_{d c}\right)\right]
$$

Controller parameters for the VSC-MTDC converter stations:

$$
K_{P}^{P}=K_{P}^{Q}=10, K_{I}^{P}=50, K_{I}^{Q}=20, k_{\text {droop }}^{V d c}=10 .
$$

VSC-MTDC converter capacitance (including lumped line capacitance) for terminals $1-6(\mathrm{pu})$ :

$$
C_{D C}^{M T D C}=\{0.1311,0.1827,0.1712,0.1770,0.1454,0.1168\}
$$

VSC-MTDC Line Data:

\begin{tabular}{ccccc}
\hline Line & From & To & $R(\mathrm{pu})$ & $L(\mathrm{pu})$ \\
\hline 1 & 1 & 2 & $5.43 \times 10^{-4}$ & $2.28 \times 10^{-5}$ \\
2 & 2 & 3 & $9.93 \times 10^{-4}$ & $4.10 \times 10^{-5}$ \\
3 & 3 & 4 & $3.31 \times 10^{-4}$ & $1.37 \times 10^{-5}$ \\
4 & 4 & 5 & $8.28 \times 10^{-4}$ & $3.41 \times 10^{-5}$ \\
5 & 4 & 6 & $2.79 \times 10^{-4}$ & $1.14 \times 10^{-5}$ \\
\hline
\end{tabular}

\section{REFERENCES}

[1] R. C. Bansal, "Bibliography on the fuzzy set theory applications in power systems (1994-2001)," IEEE Trans. Power Syst., vol. 18, no. 4, pp. 1291-1299, 2003.

[2] J. Talaq and F. Al-Basri, "Adaptive fuzzy gain scheduling for load frequency control," IEEE Trans. Power Syst., vol. 14, no. 1, pp. 145150,1999

[3] P. K. Dash, S. Mishra, and G. Panda, "Damping multimodal power system oscillation using a hybrid fuzzy controller for series connected FACTS devices," IEEE Trans. Power Syst., vol. 15, no. 4, pp. 1360 1366, 2000.

[4] R. You, H. J. Eghbali, and M. H. Nehrir, "An online adaptive neurofuzzy power system stabilizer for multimachine systems," IEEE Trans. Power Syst., vol. 18, no. 1, pp. 128-135, 2003.

[5] C.-C. Su and Y.-Y. Hsu, "Fuzzy dynamic programming: an application to unit commitment," IEEE Trans. Power Syst., vol. 6, no. 3, pp. 12311237, 1991.

[6] A. Y. Saber, T. Senjyu, T. Miyagi, N. Urasaki, and T. Funabashi, "Fuzzy unit commitment scheduling using absolutely stochastic simulated annealing," IEEE Trans. Power Syst., vol. 21, no. 2, pp. 955964, 2006.

[7] J. L. Souflis, A. V Machias, and B. C. Papadias, "An application of fuzzy concepts to transient stability evaluation [of power systems]," IEEE Trans. Power Syst., vol. 4, no. 3, pp. 1003-1009, 1989.

[8] S. K. Tso, L. Guan, Q. Y. Zeng, and K. L. Lo, "Fuzzy assessment of power system transient stability level based on steady-state data," IEE Proc. - Gener. Transm. Distrib., vol. 144, no. 6, pp. 525-531, 1997.

[9] J. M. G. Alvarez and P. E. Mercado, "Online Inference of the Dynamic Security Level of Power Systems Using Fuzzy Techniques," IEEE Trans. Power Syst., vol. 22, no. 2, pp. 717-726, 2007.

[10] J. L. Rueda, D. G. Colome, and I. Erlich, "Assessment and Enhancement of Small Signal Stability Considering Uncertainties," IEEE Trans. Power Syst., vol. 24, no. 1, pp. 198-207, 2009.

[11] C. K. Pans, Z. Y. Dong, P. Zhang, and X. Yin, "Probabilistic analysis of power system small signal stability region," in International Conference on Control and Automation. ICCA '05., 2005, vol. 1, pp. 503-509.
[12] R. Arrieta, M. A. Rios, and A. Torres, "Contingency Analysis and Risk Assessment of Small Signal Instability," Power Tech, 2007 IEEE Lausanne. pp. 1741-1746, 2007.

[13] M. A. Rios, R. Arrieta, and A. Torres, "Angular Instability 'Day Ahead' Risk Forecasting - Probabilistic Dependency on Load," IEEE Lat. Am. Trans., vol. 5, no. 8, pp. 585-590, 2007.

[14] J. Rueda and I. Erlich, "Probabilistic framework for risk analysis of power system small-signal stability," Proc. Inst. Mech. Eng. Part O J. Risk Reliab., vol. 226, no. 1, pp. 118-133, 2012.

[15] R. Preece and J. V. Milanovic, "Assessing the risk of small disturbance instability in mixed AC/DC networks," in IREP Symposium Bulk Power System Dynamics and Control IX, 2013, pp. 1-8.

[16] J. R. Hockenberry and B. C. Lesieutre, "Evaluation of Uncertainty in Dynamic Simulations of Power System Models: The Probabilistic Collocation Method," IEEE Trans. Power Syst., vol. 19, no. 3, pp 1483-1491, Aug. 2004

[17] X. Xu, T. Lin, and X. Zha, "Probabilistic analysis of small signal stability of microgrid using point estimate method," in 2009 International Conference on Sustainable Power Generation and Supply, 2009, pp. 1-6.

[18] P. Kundur, Power System Stability and Control. New York: McGrawHill, Inc., 1994.

[19] Y. Xue, T. Van Custem, and M. Ribbens-Pavella, "Extended equal area criterion justifications, generalizations, applications," IEEE Trans. Power Syst., vol. 4, no. 1, pp. 44-52, 1989.

[20] Alstom Grid, Network Protection \& Automation Guide. Alstom Grid 2011.

[21] E. H. Mamdani and S. Assilian, "An experiment in linguistic synthesis with a fuzzy logic controller," Int. J. Man. Mach. Stud., vol. 7, no. 1, pp. 1-13, Jan. 1975.

[22] B. Bede, Mathematics of Fuzzy Sets and Fuzzy Logic, vol. 295. New York: Springer Inc., 2013.

[23] R. D. Zimmerman, C. E. Murillo-Sanchez, and R. J. Thomas, "MATPOWER: Steady-State Operations, Planning, and Analysis Tools for Power Systems Research and Education," IEEE Trans. Power Syst., vol. 26, no. 1, pp. 12-19, Feb. 2011.

[24] B. Pal and B. Chaudhuri, Robust Control in Power Systems. New York: Springer Inc., 2005.

[25] G. Rogers, Power System Oscillations. Norwell: Kluwer Academic Publishers, 2000.

[26] H. F. Latorre, M. Ghandhari, and L. Söder, "Active and reactive power control of a VSC-HVdc," Electr. Power Syst. Res., vol. 78, no. 10, pp. $1756-1763$, Oct. 2008

[27] R. Preece, J. V. Milanovic, A. M. Almutairi, and O. Marjanovic, "Probabilistic Evaluation of Damping Controller in Networks With Multiple VSC-HVDC Lines," IEEE Trans. Power Syst., vol. 28, no. 1, pp. 367-376, Feb. 2013.

[28] S. Cole, J. Beerten, and R. Belmans, "Generalized Dynamic VSC MTDC Model for Power System Stability Studies," IEEE Trans. Power Syst., vol. 25, no. 3, pp. 1655-1662, Aug. 2010.

[29] J. Beerten, D. Van Hertem, and R. Belmens, "VSC MTDC Systems with a Distributed Voltage Control - A Power Flow Approach," IEEE PowerTech. Trondheim, Norway, 2011.

[30] R. Preece, A. Adrees, and J. V. Milanović, "Risk-based Framework for Assessment of Operational Constraints for Power Systems Focussing on Small-disturbance Stability and Sub-synchronous Resonance," in IEEE ISGT Europe, 2013.

[31] W. Li and R. Billinton, "Effect of bus load uncertainty and correlation in composite system adequacy evaluation," IEEE Trans. Power Syst., vol. 6 , no. 4, pp. 1522-1529, 1991.

[32] J. P. Coelingh, A. J. M. van Wijk, and A. A. M. Holtslag, "Analysis of wind speed observations over the North Sea," J. Wind Eng. Ind. Aerodyn., vol. 61, no. 1, pp. 51-69, Jun. 1996.

[33] Areva Wind GmbH, "M5000 Technical Data," 2010.

[34] M. Ali, J. Matevosyan, and J. V. Milanović, "Probabilistic assessment of wind farm annual energy production," Electr. Power Syst. Res., vol. 89, pp. 70-79, 2012.

[35] M. Ali, I.-S. Ilie, J. V. Milanovic, and G. Chicco, "Wind Farm Model Aggregation Using Probabilistic Clustering," IEEE Trans. Power Syst., vol. 28, no. 1, pp. 309-316, Feb. 2013.

[36] G. Sinden, "Characteristics of the UK wind resource: Long-term patterns and relationship to electricity demand," Energy Policy, vol. 35, no. 1, pp. 112-127, Jan. 2007. 
[37] R. Billinton and P. R. S. Kuruganty, "Probabilistic Assessment of Transient Stability in a Practical Multimachine System," IEEE Trans. Power Appar. Syst., vol. PAS-100, no. 7, pp. 3634-3641, 1981.

[38] IEEE Committee Report, "Single phase tripping and auto reclosing of transmission lines," IEEE Trans. on Power Delivery, vol. 7, no. 1, pp. 182-192, 1992.

Robin Preece (GS'10, M'13) received his BEng degree in Electrical and Electronic Engineering in 2009 and his PhD degree in 2013, both from the University of Manchester, United Kingdom. He is currently working as a Research Associate at the same institution investigating risk and uncertainty with respect to the stability of future power systems.

Jovica V. Milanović (M’95, SM'98, F'10) received his Dipl.Ing and his MSc degrees from the University of Belgrade, Yugoslavia, his PhD degree from the University of Newcastle, Australia, and his Higher Doctorate (DSc degree) from The University of Manchester, UK, all in Electrical Engineering. Currently, he is a Professor of electrical power engineering and Director of External Affairs in the School of Electrical and Electronic Engineering at The University of Manchester (formerly UMIST), UK, Visiting Professor at the University of Novi Sad, Novi Sad, Serbia and Conjoint Professor at the University of Newcastle, Newcastle, Australia. 